\title{
The Use of a Prototype MRI Safe Glidescope in a Pediatric Patient with CHARGE Syndrome: A Case Report
}

\author{
Karina Gritsenko, Myrna Kcomt, Tricia Vecchione, Tracy Straker \\ Department of Anesthesiology, Montefiore Medical Center, Bronx, USA. \\ Email: kgritsen@montefiore.org,mkcomt@montefiore.org, tstraker@montefiore.org, tvecchio@montefiore.org
}

Received August 17 ${ }^{\text {th }}, 2012$; revised April 18 ${ }^{\text {th }}, 2013$; accepted May 6 ${ }^{\text {th }}, 2013$

Copyright (c) 2013 Karina Gritsenko et al. This is an open access article distributed under the Creative Commons Attribution License, which permits unrestricted use, distribution, and reproduction in any medium, provided the original work is properly cited.

\begin{abstract}
Introduction: Charge syndrome is a very rare condition with an estimated prevalence of one in ten thousand. The care and treatment of these patients involve an interdisciplinary team. This case reports difficult airway management of a pediatric patient with CHARGE syndrome with an MRI safe glidescope. Case Presentation: A 13-year-old female with a past medical history significant for CHARGE syndrome, lumbar scoliosis, developmental delay, vitiligo, Wenckebach type AV block, intrinsic asthma and pulmonary hemosiderosis was scheduled to undergo an MRI of the spine as preoperative imaging for scoliosis corrective surgery under sedation. The patient desaturated during sedation for IV placement. After a failed attempt at sedation, the patient was then intubated safely with an MRI compatible glidescope. Conclusion: As cases increase in remote locations, it is necessary to have access to alternative devices for intubation of a difficult airway, particularly in MRI facilities where equipment must be compatible with MRI technology.
\end{abstract}

Keywords: Charge Syndrome; Glidescope; Difficult Airway; Magnetic Resonance Imaging (MRI)

\section{Introduction}

CHARGE Syndrome was first described by Dr. BD Hall in a 1979 journal paper in which 17 children born were described with multiple congenital abnormalities, including ascertained choanal atresia. Simultaneously, Dr. HM Hittner also observed 10 children with ocular colobomas, choanal atresia, congenital heart defects and hearing loss.

In 1981, Dr. RA Pagon was the first to use the acronym "CHARGE" as a non-random association of anomalies occurring together [1]. CHARGE syndrome refers to an autosomal dominant disorder consisting of coloboma of the eye (C), heart disease $(\mathrm{H})$, atresia of choanae (A), growth retardation $(\mathrm{R})$, genital hypoplasia/urinary defects (hypogonadism) (G), and ear anomalies/deafness (E). In addition, airway abnormalities including retrognathia, glossoptosis, and laryngeal paralysis have been reported in patients with this syndrome [2]. The diagnosis is confirmed clinically and by genetic testing in $60 \%$ of this population. Genetic testing demonstrates an autosomal dominant mutation of the CDD7 gene. The true incidence of CHARGE syndrome is not known, but estimates range from 0.1 - 1.2/10,000 live births.

Anesthesia management for magnetic resonance imaging (MRI) in pediatric patients can potentially be a chal- lenging task for an anesthesiologist. Most children require deep sedation due to the length of the procedure and the need for precise positioning and patient cooperation. However, general anesthesia with securing of the airway may be necessary in some circumstances. The search for airway devices that can be used safely for patients in the MRI suite continues.

We report a case of CHARGE syndrome observed in a 13-year-old female patient with a difficult airway, in whom general anesthesia was managed successfully using a MRI safe glidescope for intubation.

\section{Case Presentation}

The patient is a 13-year-old female with a past medical history of CHARGE syndrome, prematurity (ex-36 weeks), lumbar scoliosis, developmental delay, vitilgo, bradycardia (Wenchebach type AV block), mild asthma (albuterol prn), and pulmonary hemosiderosis and stenosis. Surgical history was significant for pulmonary stenosis, VSD and PDA, both repaired in infancy. The choanal atresia was also surgically repaired at 9 days of age. She has no known drug allergies.

The mother reported that as a younger child, she had a "somewhat difficult intubation" and was slow to emerge 
from anesthesia. However, she did not know how the patient was intubated in the past.

Physical Examination:

BP-101/46, HR-45-70, T-37, HT-134 cm, Wt. 28.7 kg, Sat $95 \%$;

Airway-Mallampati could not be assessed because patient was uncooperative. Exam noted thyromental distance less than $6 \mathrm{~cm}$, small nostrils, retronagthia and irregular teeth;

Heart-2/6 murmur;

Lungs-decreased breath sounds bilaterally;

Abdomen-no distension, +BS;

Lab values-all within normal limits.

A discussion with the mother included counseling regarding a potential for a difficult airway and consent was obtained. Anesthetic options included MAC, or general anesthesia with a secondary airway plan, including an MRI compatible Glidescope for potential difficult airway preparation. With mother at bedside, standard MRI compatible ASA monitors were applied. Due to lack of cooperation, a sponataneously breathing sedation was initiated with intramuscular ketamine $50 \mathrm{mg}$ and IV access was obtained to provide $2 \mathrm{mg}$ of midazolam IV. The mother was taken to the waiting room. With mild sedation, tracheal tugging was noted with each breath. Accessory muscle use and nasal flaring increased dramatically while the oxygen saturation decreased to $80 \%$ accompanied by bradycardia. An oral airway and shoulder roll were placed and positive pressure by bag mask ventilation was initiated with a good response. Atropine $0.2 \mathrm{mg}$ was given for brief heart rate was in the 40's. Oxygen saturation recovered to $100 \%$ and vital signs were stabilized.

As the patient had regained spontaneous ventilation with no obstruction and was still sedated, a continuation of MAC anesthesia was considered using the mask attached to the anesthesia circuit as the oxygen source. The mandible was pulled forward and secured with tape to the MRI gurney. A nasal airway was attempted, but was not successful as the nostrils were extremely narrow. Ten minutes into the scan, this plan was aborted when the patient began moving and crying. The scan was stopped, the patient removed from the scanner and comforted. The child was now fully awake. The decision was then made to proceed with general anesthesia.

Since controlled mask ventilation had been demonstrated, it was felt that induction of general anesthesia could proceed safely in the MRI suite with MRI-compatible glidescope for intubation due to history of unknown airway issues in the past. A second anesthesiologist and an anesthesiology tech were available to assist.

After pre-oxygenation, an inhalational induction of anesthesia with Sevoflurane was performed with maintainance of spontaneous respiration. $20 \mathrm{mg}$ of Propofol and $0.2 \mathrm{mg}$ of Atropine were administered for intubation.
The Glidescope was passed into the airway and vocal cords were visualized with significant cricoid pressure (Cormack and Lehane grade 3), but a 5.5 cuffed endotracheal tube with a Parker Flex tip stylet was not able to pass through the cords. The patient was then successfully intubated with a size 5.0 ETT with stylet in place on a second attempt. A designated anesthesiology technician was accoun.le for the stylet throughout the intubation to avoid any movement of the stylet into the MRI machine. General anesthesia was maintained with Sevoflurane 0.5 MAC under spontaneous ventilation.

The scan was uneventful and the patient was extubated without complication. Dexamethasone $8 \mathrm{mg}$ was given to prevent airway swelling and Zofran $4 \mathrm{mg}$ to prevent nausea and vomiting. The patient was discharged from the MRI post anesthesia care unit 30 minutes later.

\section{Discussion}

The ability to safely intubate a difficult airway with a Glidescope is an important and useful adjuvant technique for an anesthesiologist during the assessment of the difficult airway algorithm. As cases increase in remote locations, it is necessary to have the cooperation of the entire team and access to devices that are essential for securing a difficult airway.

During the past 10 years, there has been an explosion of intubating devices in the field of anesthesiology. None of these devices has had a larger impact than videolaryngoscopy. What was once deemed an automatic awake fiberoptic intubation may have other options for intubation. In addition, the number of procedures done outside of the operating room has increased dramatically over the years. The Glidescope is one example, which has provided benefit in clinical practice, and its role has expanded into new clinical environments, such as the MRI suite, thereby greatly enhancing patient safety.

The MRI suite can be a challenging area for the anesthesiologist. It is often remote from the operating room and additional skilled personnel who can assist when help is needed. Most children require sedation, analgesia, anxiolysis to achieve the degree of cooperation or immobilization necessary to obtain satisfactory MRI images. Moreover, deep sedation is often required in pediatric patients, which may result in upper airway obstruction in spontaneously breathing patients [3]. Failed sedation in the pediatric population has been associated with greater age, higher ASA status (III and IV), and the use of benzodiazepines [4]. This complication may necessitate general anesthesia with subsequent endotracheal intubation. A difficult airway in the pediatric patient having an MRI can be life threatening. The potential for "help to arrive" in a timely manner is critical for neonates, infants and children. The margin of safety in the maintenance of a un- 
protected airway is significantly less in children and the MRI environment creates a unique set of potential risks and limitations [3]. This population has less functional residual capacity (FRC) and higher minute ventilation than adults. The decrease in FRC limits oxygen reserves during the period of apnea/intubation and predisposes the pediatric population to atelectasis and hypoxemia.

It is in these scenarios that the MRI safe Glidescope can be helpful as an adjuvant visualization tool and may even be lifesaving. If the MRI safe Glidescope was not available for this case, the patient would have either been awakened rescheduled for another day or the patient would have been re-anesthesthetized outside of the MRI area with adjuvant equipment such as a pediatric fiberoptic scope available. The latter option would require transportation to the MRI suite under endotracheal total intravenous anesthesia (TIVA). In our hospital, a fiberoptic intubation could mean transportation of an intubated patient to the MRI suite under total intravenous anesthesia (TIVA) and transfer between floors. In this case scenario, the patient safety was maintained with a useful alternative MRI-compatible airway device available.

\section{Conclusion}

As cases increase in remote locations, it is necessary to have access to alternative devices for intubation of a dif- ficult airway, particularly in MRI facilities where equipment must be compatible with MRI technology. This case provides one example of a videolaryngoscope, which can now be used safely in the MRI suite.

\section{REFERENCES}

[1] K. D. Blake and C. Prasad, “CHARGE Syndrome,” Orphanet Journal of Rare Diseases, Vol. 1, No. 34, 2006.

[2] Y. Haha, K. Hirota and K. Fukuda, "Successful Airway Management with the Use of a Laryngeal Mask Airway in a Patient with CHARGE Syndrome,” Journal of Anesthesia, Vol. 23, No. 4, 2009, pp. 630-632. doi:10.1007/s00540-009-0791-y

[3] A. S. Matveevskii and M. Mahmoud, "New Airway Device for Ventilation and Monitoring in Pediatric Patients Undergoing MRI Study," Journal of Clinical Monitoring and Computing, Vol. 26, No. 17, 2012, p. 20.

[4] S. Malviya, T. Voepel-Lewis, O. P. Eldevik, D. T. Rockwell, J. H. Wong and A. R. Tait, "Sedation and General Anesthesia in Children Undergoing MRI and CT: Adverse Events and Outcomes," British Journal of Anesthesia, Vol. 84, No. 6, 2000, pp. 743-748. doi:10.1093/oxfordjournals.bja.a013586 